\title{
Analysis of condylar volume in relation to craniofacial morphology using cone beam computed tomography
}

\author{
Maryam Mostafavi, ${ }^{a}$ Arman Saeedi Vahdat, ${ }^{\mathrm{b}}$ Laleh Javadian, ${ }^{\mathrm{c}}$ and Aisan Ghaznavi ${ }^{\mathrm{a}^{*}}$
}

aDepartment of Oral and Maxillofacial Radiology, Dentistry faculty, Urmia University of Medical Sciences, Urmia, Iran.
bDepartment of Oral and Maxillofacial Radiology, Dentistry Faculty, Tabriz University of Medical Sciences, Tabriz, Iran.
'Department of Oral and Maxillofacial Radiology, General dentist,Dentistry Faculty, Urmia University of Medical Sciences, Urmia, Iran.
${ }^{*}$ Correspondence to Aisan Ghaznavi (email: aisanghaznavi@yahoo.com).
(Submitted: 14 September 2018 - Revised version received: 28 September 2018 - Accepted: 17 November 2018 - Published online: 26 December 2018)

\begin{abstract}
Objectives This study aimed at comparing the size and shape of mandibular condyle in mature adult population with different skeletal patterns.

Methods A total of 198 patients within the age range of 15-64 years, including 68 males and 130 females, were allocated into three groups based on the A point-nasion-B point angle ANB angle: skeletal classes I $(n=65)$, II $(n=69)$, and III $(n=64)$. The cone beam computed tomography (CBCT) was used to evaluate right and left temporomandibular joint (TMJ) in each patient. TMJ evaluation was comprised of size of condyle, area of condyle, and morphology index. The Mimics software was used to calculate the size and area of the condyle. The size, area, and morphology index were compared between the study groups using parametric tests.

Results Based on the results of paired t-test, there was a significant difference between the sizes of right and left condyles, in favor of the right condyle $(P=0.02)$. In addition, the mean size of the condyle in class III subjects was higher than those of classes I and II; however, the difference between the classes I and II was insignificant. The size and area of condyle were higher in males compared with females. Conclusion Based on the results of this study, there might be a correlation between the facial skeletal morphology and area of condyle. Keywords cone beam computed tomography, mandibular condyle, malocclusion, morphology
\end{abstract}

\section{Introduction}

The temporomandibular joint (TMJ) is one of the complex joints in the human body, which plays an essential role in many important functions, such as chewing, swallowing, and speaking. The coordination and balance of TMJ are extremely important for a normal rodent. Condyle, as an integral part of TMJ, has the capacity of remodeling in response to mandibular rotation and displacement in different directions. The morphology and volume of condyle represent the level of adaptability to functional stimuli and influence the long-term stability of orthodontic and orthognathic treatments. Moreover, condyle is important in determining the mandibular size, and condylar morphology can be associated with the position of maxillary and mandibular bases.

Previous studies have examined the relationship between TMJ morphology and malocclusion using two-dimensional (2D) images. ${ }^{2,3}$ Considering the limitations of these images, it is difficult to conduct such analyses and thoroughly examine all dimensions. However, with the advent of three-dimesional (3D) imaging modalities, such as computed tomography (CT) and cone beam computed tomography (CBCT), evaluation of condylar morphology is now possible.

Krisjane et al. ${ }^{4,5}$ morphologically evaluated TMJ in patients with classes II and III malocclusions using CT images. The results showed that condylar size was smaller in class II patients, compared with class III patients. Considering the high efficacy of CBCT in presenting 3D images without superimposition, magnification, or distortion (with limited exposure dose), researchers started to use this imaging modality for the analysis of TMJ only 2 years after its introduction to jaw and facial imaging. ${ }^{6}$

Research has been conducted on the relationship between skeletal morphology and condylar volume and morphology using CBCT. Clinicians can assess abnormal condyle morphology and potential TMJ disorders with respect to the normal range of condyle morphological parameters. ${ }^{7}$ In a previous study, Saccucci et al. ${ }^{8}$ reported a correlation between skeletal morphology and condylar volume and surface, based on the CBCT images in a Caucasian population and suggested further studies on different populations considering the racial and regional differences.

Since no study has been carried out in this area in the country where authors live, the aim of this study was to evaluate the relationship between the morphological index and condylar volume and surface in patients with classes I-III orthodontics in the Northwest of that country.

\section{Materials and Methods}

In this study, a total of 198 3D CBCT images, acquired for the evaluation of orthodontic problems, were examined in patients, aged between 15 and 64 years. Since clinical examinations had been already performed on these patients, those with clinical signs and symptoms of TMJ disorders were not recruited in the study. All images were acquired using NewTom VGI (QR, Verona, Italy) with a cone-shaped X-ray beam, a $360^{\circ}$ rotation, and a flat-panel detector (pixel size, 0.5 $\mathrm{mm}$ ). The imaging features included intermittent exposure with a maximum voltage of $120 \mathrm{kVp}$, current of 3-8 $\mathrm{mA}$, and scan time of $18 \mathrm{~s}$.

The images were evaluated by two observers (a student of dentistry and a radiologist) and observed on a 17-inch LCD VAIO screen (F-series, Japan) at a resolution of $1024 \times 1208$ in a room with medium lighting. The images were recorded in DICOM format using NNT Viewer 2.21, and then entered into Planmeca in order to determine the ANB angle. The lateral cephalometric view was derived from $\mathrm{CBCT}$ images and 
categorized into classes I-III, based on the Steiner's analysis. The patients were classified with respect to the ANB angle: class I: $2^{\circ}-4^{\circ}$; class II: $>4^{\circ}$; and class III: $<2^{\circ}$ (Fig. 1 ).

The images were entered into Mimics 10.01 for volumetric measurements after determining the ANB angles. Each condyle was reconstructed three-dimensionally using the mentioned software. First, a mask was constructed from one side of the patient, which was used to parallelize the axial planes with the Frankfurt plane (Fig. 2). Using axial sections, the upper limit of the condyle was defined where the first radiopaque area was viewed in the synovial area, while the lower limit was determined using the plane crossing the sigmoid notch.

Another mask was constructed by separating the condyle and adjacent tissues (Fig. 3). In all coronal sections with thickness of $3 \mathrm{~mm}$, areas unrelated to condyle (e.g. cortical bone, cartilage, etc.) were removed. In the axial sections, the limits of the constructed mask were determined. Finally, 3D reconstruction was done, and volumetric measurements (in $\mathrm{mm}^{2}$ ) were performed using the software (Fig. 4).

To evaluate intraoperator errors, the CBCT images of $10 \%$ of subjects were reevaluated (with at least a 1-week interval). Moreover, for determining interoperator errors, $10 \%$ of CBCT images were reevaluated by another observer.

In this study, statistical analysis was performed using SPSS version 16 (Chicago, SPSS Inc). Parametric tests, including one-way ANOVA: Analysis of Variance (is a statistical technique for testing if $3(+)$ population means are all equal). Tukey's multiple comparison test, independent sample $t$-test, and paired $t$-test, were used to determine differences in condylar volume among classes I-III patients and to evaluate gender differences.

\section{Results}

A total of 198 patients were recruited in this study, including $130(65.7 \%)$ females and $68(34.3 \%)$ males. The mean age of the patients was $33.01 \pm 0.66$ years. Based on these findings, $65(32.8 \%)$ patients had class I malocclusions, $69(34.8 \%)$

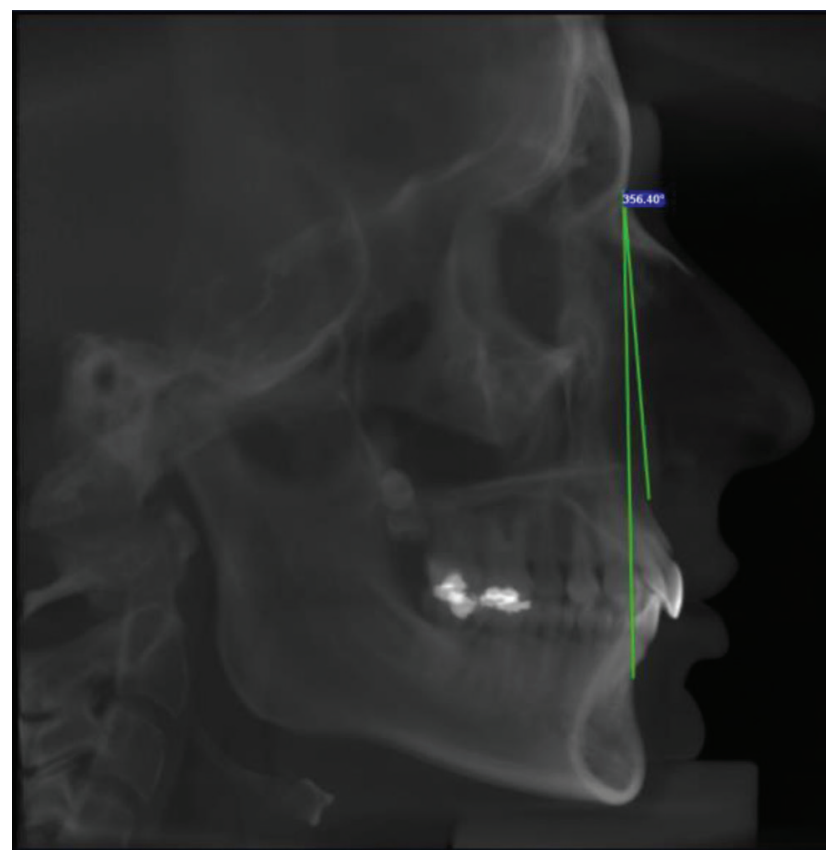

Fig. 1 The lateral cephalometric view in a CBCT image.
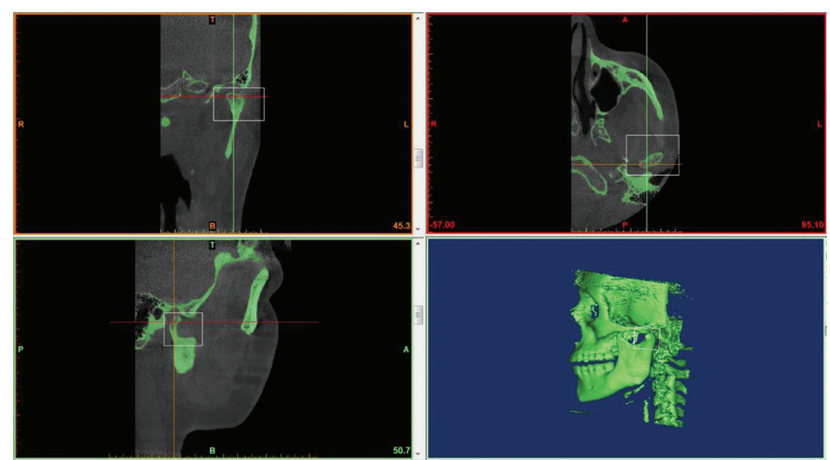

Fig. 2 The mask from one side of the patient's face.
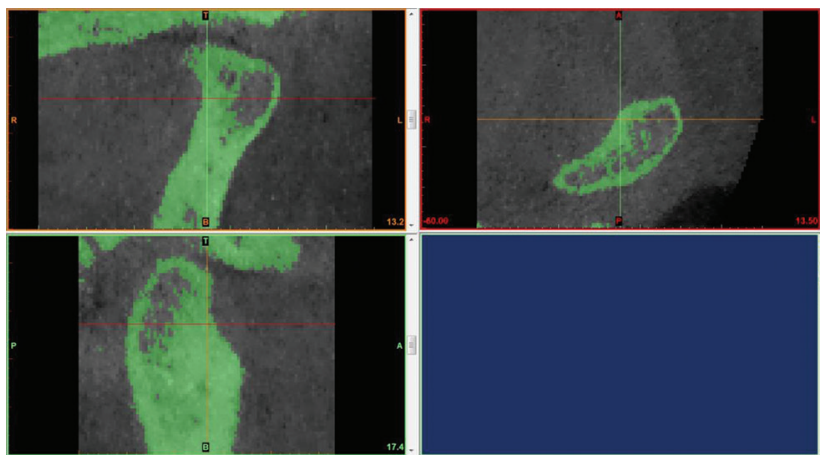

Fig. 3 The mask from the condyle and adjacent tissues.
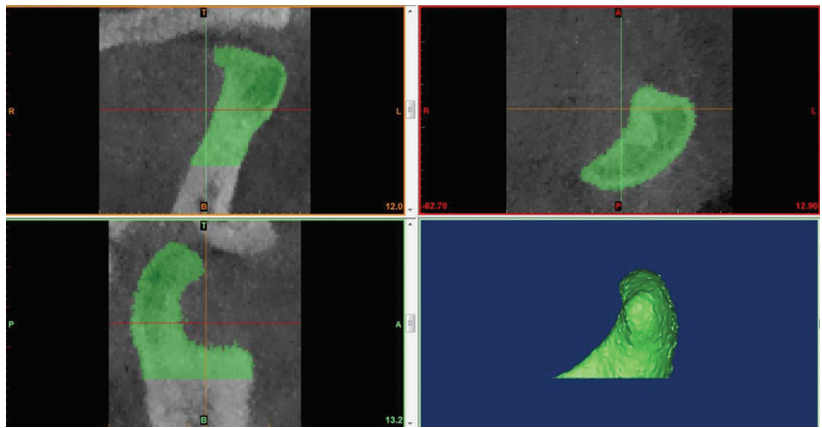

Fig. 4 The 3D image of the condyle.

patients had class II malocclusions, and $64(32.3 \%)$ patients had class III malocclusions.

Kolmogorov-Smirnov test was used to analyze the normal distribution of data. Considering the significant levels of variables ( $>0.05$ ), the zero assumption that data were not normally distributed was rejected; it was concluded that all variables have a normal distribution. Therefore, parametric tests, including one-way ANOVA, Tukey's multiple comparison test, independent sample $t$-test, and paired $t$-test, were applied.

According to one-way ANOVA, condylar volume and surface were significantly different among classes I-III patients in both right and left sides $(P=0.00)$. Moreover, the results of Tukey's multiple comparison test showed that condylar volume was significantly different on the left and right sides between classes I and III patients $(P=0.00)$, as well as classes II and III $(P=0.00)$ patients.

The results of one-way ANOVA showed that the condylar morphological index was significantly different on the left $(P=$ $0.03)$ and right $(P=0.04)$ sides in classes I-III patients. 
Moreover, the results of Tukey's multiple comparison test showed that the condylar morphological index was significantly different between the left $(P=0.03)$ and right $(P=0.02)$ sides in classes I and III patients, as well as classes II and III patients (left and right sides, $P=0.01$ ) (Table 1 ).

The mean condylar volume and surface, as well as the morphological index, were higher in class III patients, compared with classes I and II patients (Tables 2 and 3). Nevertheless, the difference was not significant between classes I and II patients. According to the intraclass correlation, or the interclass correlation coefficient (ICC), index, the interobserver agreement, as well as the agreement between the first and second observations, was very good (>93\%). Based on the independent sample $t$-test, condylar volume and surface were greater in men, compared with women $(P=0.00)$; however, the morphological index was not significantly different. The results of paired $t$-test showed that the left and right condylar volumes were significantly different, with a higher right condylar volume $(P=0.02)$; however, the condylar surface was not significantly different.

\section{Discussion}

Radiological examination of TMJ is quite challenging, since thorough evaluation is not possible with conventional radiography,

\begin{tabular}{lccccccc}
\hline \multicolumn{7}{l}{ Table 1. The post-hoc evaluation (Tukey's test) } \\
\hline $\begin{array}{c}\text { Volume } \\
\text { (L) }\end{array}$ & $\begin{array}{c}\text { Volume } \\
\text { (R) }\end{array}$ & $\begin{array}{c}\text { Surface } \\
\text { (L) }\end{array}$ & $\begin{array}{c}\text { Surface } \\
\text { (R) }\end{array}$ & $\begin{array}{c}\text { MI } \\
\text { (L) }\end{array}$ & $\begin{array}{c}\text { MI } \\
\text { (R) }\end{array}$ \\
\hline Class 1 & $P=0.73$ & $P=0.65$ & $P=0.65$ & $P=0.94$ & $P=0.80$ & $P=0.16$ \\
Class 2 & & & & & & \\
Class 1 & $P=0.00$ & $P=0.00$ & $P=0.00$ & $P=0.00$ & $P=0.03$ & $P=0.02$ \\
Class 3 & & & & & & \\
Class 2 & $P=0.00$ & $P=0.00$ & $P=0.00$ & $P=0.00$ & $P=0.01$ & $P=0.01$ \\
Class 3 & & & & & & \\
\hline
\end{tabular}

and accurate analysis requires advanced techniques. With the advent of CBCT technique, complete evaluation of the bone structure of TMJ is now possible. According to the review of literature, only one study has examined the relationship between condylar volume and surface and skeletal classes I-III, using CBCT images in a Caucasian population. ${ }^{8}$ Therefore, the aim of this study was to evaluate condylar volume and surface, as well as the morphological index in classes I-III patients in the Northwest of the country where the authors live.

In this study, the mean age of the subjects was 15-64 years due to the limited number of samples under 30 years. Since the condyle undergoes remodeling and degenerative changes with age, affecting the condylar surface and volume, only samples with a normal condylar bone structure were recruited in the study. A total of 198 3D CBCT images, acquired for orthodontic purposes, were collected from patients. Of all patients, 65 patients with the mean age of 32.5 years were classified as class I, 69 patients with the mean age of 34.8 years were classified as class II, and 64 patients with the mean age of 31.5 years were classified as class III.

The left and right condylar volume and surface were significantly higher in class III patients, compared with classes I and II patients. Furthermore, class III patients showed a significantly different morphological index, compared with classes I and II patients. However, the condylar morphological index, surface, and volume were not significant between classes I and II patients.

Saccucci et al. conducted a study to evaluate condylar volume and surface in classes I-III patients, using CBCT images from 200 subjects, aged 15-30 years. According to the results, condylar volume and surface in skeletal class III were significantly higher than skeletal classes I and II; however, these parameters were significantly lower in skeletal class II, compared with classes I and III. Moreover, the morphological index was significantly different between the skeletal classes on both sides. ${ }^{8}$ These results are similar to this study for the skeletal class III and different for classes I and II. Considering

Table 2. The mean, standard deviation, range, minimum, and maximum values of the morphological index, condylar surface, and condylar volume in men and women

\begin{tabular}{|c|c|c|c|c|c|c|}
\hline & $N$ & Mean & Std. deviation & Range & Minimum & Maximum \\
\hline \multicolumn{7}{|l|}{ Female } \\
\hline Volume (left) & 130 & 1482.12 & 301.87 & 1374.13 & 929.87 & 2304.00 \\
\hline Surface (left) & 130 & 883.41 & 125.95 & 753.95 & 613.12 & 1367.07 \\
\hline MI (left) & 130 & 0.6 & 0.06 & 0.41 & 0.5 & 0.91 \\
\hline Volume (right) & 130 & 1504.66 & 286.39 & 1387.07 & 922.97 & 2310.04 \\
\hline Surface (right) & 130 & 883.17 & 110.48 & 596.4 & 607.7 & 1204.1 \\
\hline MI (right) & 130 & 0.59 & 0.05 & 0.32 & 0.48 & 0.80 \\
\hline \multicolumn{7}{|l|}{ Male } \\
\hline Volume (left) & 68 & 1859.94 & 498.81 & 2743.45 & 913.83 & 3657.28 \\
\hline Surface (left) & 68 & 1068.83 & 210.05 & 1190.13 & 644.45 & 1834.58 \\
\hline MI (left) & 68 & 0.58 & 0.07 & 0.5 & 0.46 & 0.96 \\
\hline Volume (right) & 68 & 1874.18 & 491.43 & 2732.15 & 867.97 & 3600.12 \\
\hline Surface (right) & 68 & 1066.10 & 217.58 & 1157.5 & 643.1 & 1800.6 \\
\hline MI (right) & 68 & 0.57 & 0.05 & 0.031 & 0.46 & 0.77 \\
\hline
\end{tabular}

For volume/surface (independent sample $t$-test), $P=0$; For right IM (independent sample $t$-test), $P=0.59$; For left IM (independent sample $t$-test), $P=0.22$. 
Table 3. The mean \pm SD of morphological index, condylar surface, and condylar volume in men and women in the left and right condyles

\begin{tabular}{lcccc}
\hline & \multicolumn{2}{c}{ Left } & \multicolumn{2}{c}{ Right } \\
\cline { 2 - 5 } & Mean & Std. deviation & Mean & Std. deviation \\
\hline Volume & 1609.99 & 419.05 & 1629.76 & 407.15 \\
Surface & 946.18 & 181.63 & 945.09 & 177.33 \\
Ml & 0.6 & 0.06 & 0.59 & 0.059 \\
\hline
\end{tabular}

For volume (paired $t$-test), $P=0.02$; For surface (paired $t$-test), $P=0.83$.

the similar sample size and methods, the differences might be due to racial differences.

In this study, the mean condylar volume in classes I-III patients were respectively $1545.48,1515.44$, and 1859.62 $\mathrm{mm}^{2}$ on the right side and $1524.45,1500.95$, and 1835.58 $\mathrm{mm}^{2}$ on the left side. However, in the study by Saccucci et al. the condylar volume in classes I-III patients were respectively $2693.09,2350.64$, and $2672.80 \mathrm{~mm}^{2}$ on the right side and $2675.09,2352.02$, and $2792.78 \mathrm{~mm}^{2}$ on the left side. Despite the use of similar methods, the difference in the mean condylar volume and surface was significant between these populations.

In a previous study, Al-koshab et al. aimed to assess condylar morphology and glenoid fossa in a Southeast Asian population. The mean condylar volume, regardless of the skeletal class, was 1460.69 and 1450.89 on the right and left sides, respectively. The overall difference in condylar volume in these studies may indicate the effects of regional and racial factors, which can be evaluated in various studies on different populations.

Moreover, Katsavrias et al. reviewed 189 patients using submentovertex radiography. The results showed that the main components of condylar shape were different among skeletal classes, and the condylar head was longer in class III. ${ }^{10}$ In another study performed in 2016, Alhammadi et al. reviewed the 3D CBCT images of TMJ from 60 patients (aged 18-25 years) with skeletal classes I-III. Overall, 20 patients were assigned to class I, 20 patients were assigned to class II, and 20 patients were assigned to class III. According to the results, the lowest condylar width and the highest condylar height were reported in class II patients. ${ }^{11}$

According to the results of this study, condylar volume and surface were greater in class III patients, compared with the classes I and II. This finding may be attributed to two major factors. First, according to the Moss's functional matrix theory, growth of each skeletal unit in the maxillofacial surface is defined based on the functional requirements of the corresponding tissue. Since class III patients show larger mandibular volume, the functional requirements of the mandibular unit, and consequently condylar volume, are higher in these patients. ${ }^{12}$ A study by Enomoto et al. ${ }^{13}$ on mice showed that type of diet is effective in condylar volume. However, this finding cannot be fully accepted, as the biting force is not applied continuously, and the parafunctional habits are probably more effective than diet in condylar volume in classes I and II malocclusions.

Second, growth hormones play a very important role in the linear and angular size of craniofacial structures. Abundance or shortage of growth hormones can cause disproportionate growth in the base of skull and jaws and decrease the anterior face height, compared with the posterior face height. Considering the effects of growth hormone receptors on the growth of condyle, it can be concluded that higher condylar volume in class III patients can be due to its excessive response to growth hormones.

Moreover, in studies conducted in East Asia on patients with mandibular prognathism, there were mutations on some loci of chromosomes 1, 6, and 19. According to these findings, despite the irrefutable effects of environment, genetics play an undeniable role in mandibular growth and morphology, i.e., mandibular size, length, and height. In this study, condylar volume and surface on both the sides were significantly higher in men than women. However, the morphological index was not significantly different on either sides between males and females.

In 2010, Tecco et al. conducted a study on a Caucasian population, using CBCT images and Mimics software. The results regarding the difference in condylar morphological index and volume between men and women are consistent with the findings of the present study, which showed significantly higher values in men, compared with women. However, the difference in terms of condylar surface was not significant among men and women. ${ }^{14}$ In this regard, Al-koshab et al. ${ }^{9}$ showed that condylar volume, width, height, and shared space were significantly higher in men than women from East Asia.

According to a study by Saccucci et al., ${ }^{8}$ the condylar surface and volume were larger in men, compared with women. Moreover, Gomes et $\mathrm{al}^{15}$ showed that condylar dimensions were larger in men; the results of these studies are consistent with the present study. In addition, in our study, the right and left condylar surfaces were not significantly different. However, there was a significant difference between the right and left condylar volumes (the left condylar volume was larger). On the other hand, the morphological index was not significantly different between the right and left sides.

Krisjane et al. ${ }^{4}$ showed a significant difference in condylar height between the right and left condyles in patients with condylar asymmetry. Consistent with the present study, Tecco et al. ${ }^{14}$ showed that the right condylar volume was larger than the left condylar volume, which can be due to the similar sample size and methods of these studies. On the other hand, in the study by Saccucci et al., ${ }^{8}$ the difference between the right and left condylar volume was not significant, which is contrary to the present results, as well as studies by Krisjane and Tecco.

\section{Conclusion}

The results of this study showed differences in condylar volume and surface among classes I-III patients. Therefore, by performing similar studies on different populations, these findings can be applicable in the assessment of orthodontic problems.

\section{Conflict of Interest}

None. 


\section{References}

1. Saccucci M, Polimeni A, Festa F, Tecco S. Do skeletal cephalometric characteristics correlate with condylar volume, surface and shape? A 3D analysis. Head Face Med. 2012;8:15

2. Gianelly AA, Petras JC, Boffa J. Condylar position and class II deep-bite, nooverjet malocclusions. Am J Orthod Dentofacial Orthop. 1989;96:428-432.

3. Widman DJ. Functional and morphologic considerations of the articular eminence. Angle Orthod. 1988;58:221-236.

4. Krisjane Z, Urtane I, Krumina G, Bieza A, Zepa K, Rogovska I. Condylar and mandibular morphological criteria in the 2D and 3D MSCT imaging for patients with Class II division 1 subdivision malocclusion. Stomatologija. 2007;9:67-71.

5. Krisjane Z, Urtane I, Krumina G, Zepa K. Three-dimensional evaluation of TMJ parameters in Class II and Class III patients. Stomatologija. 2009;11:32-36.

6. Larheim TA, Abrahamsson AK, Kristensen M, Arvidsson LZ. Temporomandibular joint diagnostics using CBCT. Dentomaxillofac Radiol. 2014:44:20140235.

7. Song Y, Zhang X, Gao Y, Hou F, Yu Y. The condylar morphology in adult females of skeletal class II division 1 malocclusion with various vertical skeletal features: a study by cone beam computed tomography. Int J Clin Exp Med. 2016;9:8304-8311.

8. Saccucci M, D'Attilio M, Rodolfino D, Festa F, Polimeni A, Tecco S. Condylar volume and condylar surface in class I, class II and class III young adult subjects. Head Face Med. 2012;8:34.
9. Al-koshab M, Nambiar P, John J. Assessment of condyle and glenoid fossa morphology using CBCT in South-East Asians. PLoS One. 2015; 10:e0121682.

10. Katsavrias EG, Halazonetis DJ. Condyle and fossa shape in Class II and Class III skeletal patterns: a morphometric tomographic study. Am J Orthod Dentofacial Orthop. 2005;128:337-346. doi:10.1016/j. ajodo.2004.05.024

11. Alhammadi MS, Fayed MS, Labib A. Three-dimensional assessment of temporomandibular joints in skeletal Class I, Class II, and Class III malocclusions: cone beam computed tomography analysis. J World Fed Orthod. 2016;5:80-86

12. Moss ML, Rankow RM. The role of the functional matrix in mandibular growth. Angle Orthod. 1968;38:95-103.

13. Enomoto A, Watahiki J, Yamaguchi T, Irie T, Tachikawa T, Maki K. Effects of mastication on mandibular growth evaluated by microcomputed tomography. Eur J Orthod. 2010;32:66-70.

14. Tecco S, Saccucci M, Nucera R, Polimeni A, Pagnoni M, Cordasco G, et al. Condylar volume and surface in Caucasian young adult subjects. BMC Med Imaging. 2010;10:28.

15. Gomes AF, Nejaim Y, Brasil DM, Groppo FC, Ferreira Caria PH, Haiter Neto F. Assessment of volume and height of the coronoid process in patients with different facial types and skeletal classes: a cone-beam computed tomography study. J Oral Maxillofac Surg. 2015;73:1395.e1-e5. doi:10.1016/j.joms.2015.02.020

This work is licensed under a Creative Commons Attribution-NonCommercial 3.0 Unported License which allows users to read, copy, distribute and make derivative works for non-commercial purposes from the material, as long as the author of the original work is cited properly. 